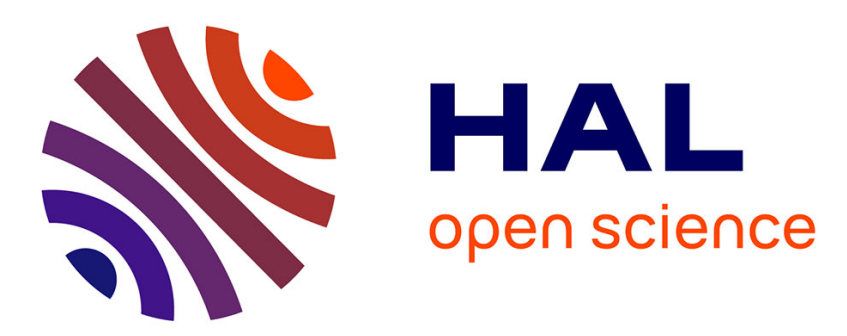

\title{
The Corona Problem on a complete ultrametric algebraically closed field
}

\author{
Alain Escassut
}

\section{To cite this version:}

Alain Escassut. The Corona Problem on a complete ultrametric algebraically closed field. p-Adic Numbers, Ultrametric Analysis and Applications, 2016. hal-01918227

\section{HAL Id: hal-01918227 https://hal.uca.fr/hal-01918227}

Submitted on 10 Nov 2018

HAL is a multi-disciplinary open access archive for the deposit and dissemination of scientific research documents, whether they are published or not. The documents may come from teaching and research institutions in France or abroad, or from public or private research centers.
L'archive ouverte pluridisciplinaire HAL, est destinée au dépôt et à la diffusion de documents scientifiques de niveau recherche, publiés ou non, émanant des établissements d'enseignement et de recherche français ou étrangers, des laboratoires publics ou privés. 


\title{
The Corona Problem on a complete ultrametric algebraically closed field
}

\author{
by Alain Escassut
}

\begin{abstract}
Let $\mathbb{K}$ be a complete ultrametric algebraically closed field and let $A$ be the Banach $\mathbb{K}$-algebra of bounded analytic functions in the "open" unit disk $D$ of $\mathbb{K}$ provided with the Gauss norm. Let $\operatorname{Mult}(A, \|$. \|) be the set of continuous multiplicative semi-norms of $A$ provided with the topology of simple convergence, let $\operatorname{Mult}_{m}(A, \|$. $\|)$ be the subset of the $\phi \in \operatorname{Mult}(A, \|$. $\|)$ whose kernel is a maximal ideal and let $\operatorname{Mult}_{1}(A, \|$. \|) be the subset of the $\phi \in \operatorname{Mult}(A, \|$. $\|)$ whose kernel is a maximal ideal of the form $(x-a) A$ with $a \in D$. By analogy with the Archimedean context, one usually calls ultrametric Corona problem the question whether $\operatorname{Mult}_{1}(A, \|$. $\|)$ is dense in $\operatorname{Mult}_{m}(A, \|$. \|). In a previous paper, it was proved that when $\mathbb{K}$ is spherically complete, the answer is yes. Here we generalize this result to any algebraically closed complete ultrametric field, which particularly applies to $\mathbb{C}_{p}$.

On the other hand, we also show that the continuous multiplicative semi-norms whose kernel are neither a maximal ideal nor the zero ideal, found by Jesus Araujo, also lie in the closure of $\operatorname{Mult}_{1}(A, \|$. $\|)$, which suggest that $\operatorname{Mult}_{1}(A, \|$. $\|)$ might be dense in $\operatorname{Mult}(A, \|$. $\|)$.
\end{abstract}

2000 Mathematics subject classification: Primary 12J25 Secondary 46S10

Keywords: p-adic analytic functions, corona problem, multiplicative spectrum

\section{Introduction and results.}

Let $E$ be the open disk of center 0 and radius 1 in $\mathbb{C}$ and let $B=H^{\infty}(E)$ be the unital Banach algebra of bounded analytic functions on $E$. Each point $a \in E$ defines a multiplicative linear functional $\phi_{a}$ on $B$ by $\phi_{a}(f)=f(a)$. But the set of maximal ideals of $B$ defined by points of $E$ are not the only maximal ideals of $B$. The Corona Conjecture posed by Kakutani in 1941 stated that the set of maximal ideals defined by points of $E$ is dense in the whole set $\operatorname{Max}(B)$ of maximal ideals with respect to the Gelfand topology which is the topology of pointwise convergence on $B$, defined on the space $\operatorname{Max}(B)$. This was famously proved by Carleson in 1962 [4]. The key fact is that if $f_{1}, \ldots, f_{n}$ belong to $B$ and if there exists $d>0$ such that, for all $z \in D$ we have $\left|f_{1}(z)\right|+\ldots \ldots+\left|f_{n}(z)\right|>d$ then the ideal generated by the $f_{1}, \ldots, f_{n}$ is the whole algebra $B$. People often transfer the name "Corona Statement" to this key fact. Indeed, this Corona Statement implies that the Corona Conjecture is true, thanks to the fact that all maximal ideals of a Banach $\mathbb{C}$-algebra are of codimension 1.

Now consider the situation in the non-Archimedean context. Let $\mathbb{I K}$ be an algebraically closed field complete with respect to an ultrametric absolute value $\mid$. |. Given $a \in \mathbb{K}$ and $r>0$, we denote by $d(a, r)$ the disk $\{x \in \mathbb{K}|| x-a \mid \leq r\}$, by $d\left(a, r^{-}\right)$the disk 
$\{x \in \mathbb{K}|| x-a \mid<r\}$, by $C(a, r)$ the circle $\{x \in \mathbb{K}|| x-a \mid=r\}$ and set $D=d\left(0,1^{-}\right)$. Let $A$ be the $\mathbb{K}$-algebra of bounded power series converging in $D$ which is complete with respect to the Gauss norm defined as $\left\|\sum_{n=1}^{\infty} a_{n} x^{n}\right\|=\sup _{n \in \mathbb{N}}\left|a_{n}\right|$. We know that this norm actually is the norm of uniform convergence on $D$ [8], Theorem 13.9 or [15].

In [19] the Corona problem was considered in a similar way as it is on the field $\mathbb{C}$ [4]: the author asked the question whether the set of maximal ideals of $A$ defined by the points of $D$ (which are well known to be of the form $(x-a) A$ ) is "dense" in the whole set of maximal ideals with respect to a so-called "Gelfand Topology". In fact, the Gelfand topology was originally defined on a Banach algebra $B$ on the field $\mathbb{C}$ where all maximal ideals are known to be of codimension 1 and hence each one is the kernel of a $\mathbb{C}$-algebra morphism onto $\mathbb{C}$. Therefore the pointwise topology on the set of $\mathbb{C}$-algebra morphisms from $B$ to $\mathbb{C}$ is called the Gelfand topolgy. A similar topology exists on a Banach $\mathbb{I K}$ algebra when all maximal ideals have codimension 1. But as explained in [10], this makes no sense when certain maximal ideals are of infinite codimension, which is the case for our algebra $A$, since the maximal ideals which are not of the form $(x-a) A$ are of infinite codimension [10] and therefore, there is no Gelfand topology on the whole set of maximal ideals of $A$. Consequently, a Corona problem should be defined in a different way, as explained in [10]. However, in [19] a "Corona Statement" similar to that mentioned above was shown in our algebra $A$ and it is useful in the present paper as it was in [10].

Given a commutative unital $\mathbb{K}$-algebra $B$, provided with a $\mathbb{K}$-algebra norm $\|$. $\|$, the set of continuous multiplicative $\mathbb{K}$-algebra semi-norms of $B$ was studied in many works [2], [7], [8], [9], [14] and is usually denoted by $\operatorname{Mult}(B, \|$. \|) [7], [8], [9], [14]. For each $\phi \in \operatorname{Mult}(B, \|$. $\|)$, we denote by $\operatorname{Ker}(\phi)$ the closed prime ideal of the $f \in B$ such that $\phi(f)=0$. The set of the $\phi \in \operatorname{Mult}(B,\|\|$.$) such that \operatorname{Ker}(\phi)$ is a maximal ideal is denoted by $\operatorname{Mult}_{m}(B,\|\|$.$) and the set of the \phi \in \operatorname{Mult}(B,\|\|$.$) such that \operatorname{Ker}(\phi)$ is a maximal ideal of codimension 1 is denoted by $\operatorname{Mult}_{1}(B,\|\|$.$) .$

We know that $\sup \{\phi(f) \mid \phi \in \operatorname{Mult}(B,\|\|)\}=.\lim _{n \rightarrow \infty}\left(\left\|f^{n}\right\|\right)^{\frac{1}{n}} \forall f \in B$ [9], [13]. On the other hand, $\operatorname{Mult}(B,\|\cdot\|)$ is provided with the topology of pointwise convergence and is compact with respect to this topology [9], [14].

We know that for every $\mathcal{M} \in \operatorname{Max}(B)$, there exists at least one $\phi \in \operatorname{Mult}_{m}(B,\|\|$. such that $\operatorname{Ker}(\phi)=\mathcal{M}$ but in certain cases, there exist infinitely many $\phi \in \operatorname{Mult}_{m}(B,\|\|$. such that $\operatorname{Ker}(\phi)=\mathcal{M}$ [6], [7], [9]. A maximal ideal $\mathcal{M}$ of $B$ is said to be univalent if there is only one $\phi \in \operatorname{Mult}_{m}(B, \|$. $\|)$ such that $\operatorname{Ker}(\phi)=\mathcal{M}$ and the algebra $B$ is said to be multbijective if every maximal ideal is univalent (so, unital non-multbijective commutative Banach $\mathbb{K}$-algebras do exist).

Thus, the ultrametric Corona problem may be viewed at two levels:

1) Is $\operatorname{Mult}_{1}(A,\|\|$.$) dense in \operatorname{Mult}_{m}(A,\|\|$.$) (with respect to the topology of pointwise$ convergence)?

2) Is $\operatorname{Mult}_{1}(A, \|$. \|) dense in $\operatorname{Mult}(A, \|$. \|) (with respect to the same topology )? Actually, this way to set the Corona problem on an ultrametric field is not really 
different from the original problem once considered on $\mathbb{C}$ because on a commutative unital $\mathbb{C}$-Banach algebra, all continuous multiplicative semi-norms are known to be of the form $|\chi|$ where $\chi$ is a character of $A$. Thus the Corona problem was equivalent to show that the set of multiplicative semi-norms defined by the points of the open disk was dense inside the whole set of continuous multiplicative semi-norms, with respect to the topology of pointwise convergence.

Here we will restrict to the first level: $\operatorname{Is} \operatorname{Mult}_{1}\left(A, \|\right.$. \|) dense in $\operatorname{Mult}_{m}(A, \|$. \|) (with respect to the topology of pointwise convergence)?

The answer to that question is immediate if $A$ is multbijective. This is the case when the field $\mathbb{K}$ is strongly valued i.e. either the value group or the residue class field, or the two both are not countable [7], [9] and particularly this applies to the Levi-Civita field $\mathcal{C}$ [] that is algebraically closed, complete and has a residue field isomorphic to $\mathbb{C}$.

Recall that the field $\mathbb{K}$ is said to be spherically complete if every decreasing sequence of disks has a non-empty intersection. Then the answer to the question was given in [11] when the field is spherically complete. The gap to generalize to any complete algebraically closed field was due to Lazard's problem appearing when the field $\mathbb{K}$ is not spherically complete [16]. The problem is the following. If $\mathbb{I K}$ is spherically complete, M. Lazard proved in [16] that given any sequence $\left(a_{n}\right)_{n \in \mathbb{N}}$ such that $\left|a_{n}\right|<1$ and $\lim _{n \rightarrow+\infty}\left|a_{n}\right|=1$ and any sequence of integers $\left(q_{n}\right)_{n \in \mathbb{N}}$, there exists a function $f \in A$ admitting each $a_{n}$ for zero of order $q_{n}$. But if $\mathbb{K}$ is not spherically complete, there are counter-examples showing that such functions $f$, sometimes do not exist. In the proof of the following Proposition 11, we will need a certain function admitting for zeros a certain sequence of zeros in $D$, which requires to work in a spherically complete field.

However, the field $\mathbb{K}$ admits a spherical competion $\widehat{K}$ (that is algebraically closed). The problem is then to show that the solution we obtain on this spherical $\widehat{K}$ lets us find a similar solution on the field $\mathbb{K}$, by using a closed subspace of $\widehat{K}$ that is of countable type.

Here we will show that we can solve that problem thanks to a specific result due to Banachic properties. So, we will recall the main points of the proof of [11] and we will generalize the proof to any algebraically closed field complete with respect to an ultrametric absolute value: the first interest of such a generalization is to apply to fields such as $\mathbb{C}_{p}$, the completion of an algebraic closure of $\mathbb{Q}_{p}$. The main tools to solve this problem are the ultrametric holomorphic functional calculus [7], [9] and a Banachic property [19].

Remark Given a filter $\mathcal{G}$, if for every $f \in A,|f(x)|$ admits a limit $\varphi_{\mathcal{G}}(f)$ along $\mathcal{G}$, the function $\varphi_{\mathcal{G}}$ obviously belongs to $\operatorname{Mult}(A,\|\|$.$) . Moreover, it clearly belongs to the closure$ of $\operatorname{Mult}_{1}(A,\|\|$.$) . Consequently, if we can prove that every element of \operatorname{Mult}_{m}(A, \|$. $\|)$ is of the form $\varphi_{\mathcal{G}}$, with $\mathcal{G}$ a certain filter on $D$, Question 1) is solved. And similarly, if we could prove that every element of $\operatorname{Mult}(A, \|$. $\|)$ were of the form $\varphi_{\mathcal{G}}$, Question 2) would also be solved.

Definitions and notation: Given $a \in D$ and $r, s \in] 0,1[$ such that $r<s$, let $\Gamma(a, r, s)=$ $\{x \in \mathbb{K} r<|x-a|<s\}$. Let $\mathcal{W}$ be the filter admitting for basis the family of annuli $\Gamma(0, r, 1)$. 
Given $a \in \mathbb{K}$ and $r>0$ we call circular filter of center $a$ and diameter $R$ on $D$ the filter $\mathcal{F}$ which admits as a generating system the family of $\operatorname{sets} \Gamma\left(\alpha, r^{\prime}, r^{\prime \prime}\right) \cap D$ with $\alpha \in d(a, R), r^{\prime}<R<r^{\prime \prime}$, i.e. $\mathcal{F}$ is the filter which admits as a basis the family of sets of the form $D \cap\left(\bigcap_{i=1}^{q} \Gamma\left(\alpha_{i}, r_{i}^{\prime}, r_{i}^{\prime \prime}\right)\right)$ with $\alpha_{i} \in d(a, R), r_{i}^{\prime}<R<r_{i}^{\prime \prime} \quad(1 \leq i \leq q, q \in \mathbb{N})$.

If the field $\mathbb{K}$ is not spherically complete, we must also define circular filters with no center: given a decreasing sequence of disks $\left(D_{n}\right)$ with empty intersection, we call circular filter with no center, of basis $\left(D_{n}\right)$ the filter admitting that sequence $\left(D_{n}\right)$ for basis.

Given a filter $\mathcal{F}$ on $D$, we denote by $\mathcal{J}(\mathcal{F})$ the ideal of the $f \in A$ such that $\lim _{\mathcal{F}} f(x)=0$.

Every ultrafilter $\mathcal{U}$ on $D$ defines an element $\varphi_{\mathcal{U}}$ of $\operatorname{Mult}(A,\|\cdot\|)$ as $\varphi_{\mathcal{U}}(f)=\lim _{\mathcal{U}}|f(x)|$ : such a limit does exist because each function $f \in A$ is bounded and therefore $|f(x)|$ takes values in the compact $[0,\|f\|]$.

An ultrafilter $\mathcal{U}$ on $D$ is said to be coroner if it is thinner than $\mathcal{W}$.

A maximal ideal $\mathcal{M}$ of $A$ is said to be coroner if there exists a coroner ultrafilter $\mathcal{U}$ such that $\mathcal{M}=\mathcal{J}(\mathcal{U})$.

An element $f \in A$ is said to be quasi-invertible if it has finitely many zeros.

Given a closed bounded subset $S$ of $\mathbb{K}$, we denote by $H(S)$ the Banach $\mathbb{K}$-algebra of analytic elements on $S$, i.e. the set of limits of all sequences of rational functions with no pole in $S$ with respect to the uniform convergence on $S$ [15], [8].

Given a circular filter $\mathcal{F}$ on a disk $L$, for every $f \in H(L),|f(x)|$ admits a limit $\varphi_{\mathcal{F}}$ along $\mathcal{F}$ [12], [14], [8]. Particularly, if $\mathcal{F}$ is the filter of center $a$ and diameter $r$, we put $\varphi_{a, r}=\varphi_{\mathcal{F}}$ and let $\varphi_{a}$ be the multiplicative semi-norm defined as $\varphi_{a}(f)=|f(a)|, f \in A$. Then given an ultrafilter $\mathcal{U}$ thinner than a circular filter on $D$, of diameter $r<1$, the limit of $|f(x)|$ on $\mathcal{U}$ equals that on $\mathcal{F}$ because given $f \in A, a \in D$ and $r \in[0,1[, f$ belongs to $H(d(a, r))$ and hence $\varphi_{a, r}$ applies to $f$ and has continuation to an element of $\operatorname{Mult}(A, \|$. \|) because every function $f \in A$ belongs to $H(d(a, r))$. The situation is completely different for the circular filter $\mathcal{W}$ because many functions $f \in A$ do not belong to $H(D)$. As a consequence, the restriction of the Gauss norm defined on $A$ to $\mathbb{K}[x]$ admits many extensions on $A$, defined by various coroner ultrafilters. For example, if $f$ admits a sequence of zeros $\left(\alpha_{n}\right)\left(\lim _{n \rightarrow+\infty}\left|\alpha_{n}\right|=1\right)$, then given an ultrafilter $\mathcal{U}$ thinner than that sequence, we have $\varphi_{\mathcal{U}}(f)=0$, but of course $\|f\|>0$.

The following Theorem A derives from the general characterization of continuous multiplicative semi-norms on algebras of analytic elements [8], [12], [14]. However, here we have to consider other continuous multiplicative semi-norms because the algebra $A$ is much bigger than the algebra of analytic elements in $D$.

Theorem A [10], [11]: For every ultrafilter $\mathcal{F}$ on $D$, (respectively for every circular filter $\mathcal{F}$ of diameter $r<1$ ), on $D$, for every element $f \in A,|f(x)|$ admits a limit along $\mathcal{F}$ which belongs to $\operatorname{Mult}(A,\|\|$.$) .$ 
Thus, the question arising here is the continuation to $A$ of the Gauss norm defined on $\mathbb{I K}[x]$. The problem, then is not this simple: we have to consider coroner ultrafilters.

By Theorem (3.2) in [17], we have the following Theorem B also called Corona statement [17]:

Theorem B: Let $f_{1}, \ldots, f_{q} \in A$ satisfy $\left\|f_{j}\right\|<1 \forall j=1, \ldots, q$ and $\inf \left\{\max _{j=1, \ldots, q}\left(\left|f_{j}(x)\right|\right) \mid x \in D\right\}=\omega>0$. There exist $g_{1}, \ldots, g_{q} \in A$ such that $\sum_{j=1}^{q} g_{j} f_{j}=1$ and $\max _{j=1, \ldots, q}\left\|g_{j}\right\|<\omega^{-2}$.

Corollary B1: Let $\mathcal{I}$ be an ideal of $A$. There exists a filter $\mathcal{F}$ on $D$ such that $\mathcal{I} \subset \mathcal{J}(\mathcal{F})$.

Corollary B2: $\quad$ Let $\mathcal{M}$ be a maximal ideal of $A$. There exists an ultrafilter $\mathcal{U}$ on $D$ such that $\mathcal{M}=\mathcal{J}(\mathcal{U})$.

Theorem $\mathrm{C}$ is classical and was given in [10], [11].

Theorem C: Let $\mathcal{M}$ be a maximal ideal of $A$. Either $\mathcal{M}$ is of codimension 1 and then it is of the form $(x-a) A(a \in D)$, or it is of infinite codimension and then it is coroner, of the form $\mathcal{J}(\mathcal{U})$. Moreover, if $\mathcal{J}(\mathcal{U})$ is of infinite codimension, then:

i) $\varphi_{\mathcal{U}}$ belongs to the closure of $\operatorname{Mult}_{1}(A,\|\|$.$) .$

ii) For every $f \in \mathcal{M}, f$ is not quasi-invertible.

Remark: Characterizing the coroner ultrafilters $\mathcal{U}$ such that $\mathcal{J}(\mathcal{U})$ is a maximal ideal appears very hard. For instance, let $\mathcal{Y}$ be the filter admitting for basis the family of sets $\Gamma(0, r, 1) \backslash\left(\bigcup_{n=1}^{\infty} d\left(a_{n},\left|a_{n}\right|^{-}\right)\right)$with $a_{n} \in D, \lim _{n \rightarrow+\infty}\left|a_{n}\right|=1$ and consider an ultrafilter $\mathcal{U}$ thinner than $\mathcal{Y}$. It is a coroner ultrafilter. But $\mathcal{J}(\mathcal{U})=\{0\}$. Indeed, suppose a nonidentically zero function $f$ lies in $\mathcal{J}(\mathcal{U})$. Let $\left(a_{n}\right)$ be its sequence of zeros, set $r_{n}=\left|a_{n}\right|, n \in$ $\mathbb{N}$, and let $E=D \backslash \bigcup_{n=0}^{\infty} d\left(a_{n}, r_{n}^{-}\right)$. Clearly $|f(x)|=|f|(|x|) \forall x \in E$ hence $\lim _{\mathcal{U}}|f(x)|=$ $\|f\|$. However, $E$ belongs to $\mathcal{Y}$ and therefore, $\mathcal{U}$ is secant with $E$, a contradiction with the hypothesis $f \in \mathcal{J}(\mathcal{U})$.

However, it is obvious that maximal ideals of infinite codimension do exist. Consider a sequence $\left(a_{n}\right)_{n \in \mathbb{N}}$ such that $\lim _{n \rightarrow+\infty}\left|a_{n}\right|=1$ and $\prod_{n \in \mathbb{N}}\left|a_{n}\right|>0$ and let $\mathcal{I}$ be the ideal of $f \in A$ such that $\lim _{n \rightarrow+\infty} f\left(a_{n}\right)=0$. Then by Theorem 25.5 in [8], $\mathcal{I}$ is not $\{0\}$. But clearly, it is not included in any maximal ideal of the form $(x-a) A$. Consequently, it is included in a maximal ideal of infinite codimension.

On the other hand, the mapping $\mathcal{J}$ from the set of coroner ultrafilters to the set of ideals of $A$ is not injective. Two ultrafilters $\mathcal{U}, \mathcal{V}$ are said to be contiguous if for every $\epsilon>0$, 
there exists $X \in \mathcal{U}$ and $Y \in \mathcal{V}$ such that the distance from $X$ to $Y$ is less than $\epsilon$. Then, as noticed in [10], two contiguous coroner ultrafilters define the same ideal. Conversely, if two coroner ultrafilters $\mathcal{U}, \mathcal{V}$ define the same ideal, are they contiguous? The answer seems unclear.

In [10] and [11] we proved that there exist no continuous multiplicative norm on $A$, other than the Gauss norm, inducing the Gauss norm on $\mathbb{I K}[x]$. However, how we just saw, each coroner maximal ideal, of the form $\mathcal{J}(\mathcal{U})$, defines an element $\varphi_{\mathcal{U}}$ of $\operatorname{Mult}(A, \|$. $\|)$ whose restriction to $\mathbb{K}[x]$ is the Gauss norm, but of course $\varphi_{\mathcal{U}}$ is not the Gauss norm on certain non-quasi-invertible elements of $A$.

Theorem D is given in [10], [11].

Theorem D: Suppose A is multbijective. Then for every $\phi \in \operatorname{Mult}_{m}(A,\|\|.) \backslash M u l t_{1}(A,\|\|$. there exists a coroner ultrafilter $\mathcal{U}$ such that $\phi=\varphi \mathcal{U}$. Moreover $\operatorname{Mult}_{1}(A, \|$. $\|)$ is dense in $\operatorname{Mult}_{m}(A, \|$. \|).

In [10] and [11] we considered the following conjectures: $A$ is multbijective no matter what the complete algebraically closed field $\mathbb{K}$. We are now able to prove that conjecture.

Theorem 1: A is multbijective.

Corollary 1.1: For every $\phi \in \operatorname{Mult}_{m}(A,\|\cdot\|) \backslash \operatorname{Mult}_{1}(A,\|\cdot\|)$ there exists a coroner ultrafilter $\mathcal{U}$ such that $\phi=\varphi_{\mathcal{U}}$.

Corollary 1.2: $\operatorname{Mult}_{1}(A,\|\|$.$) is dense in \operatorname{Mult}_{m}(A,\|\|$.$) .$

Remark: Thus we have proved that every element of $\operatorname{Mult}_{m}(A, \|$. $\|)$ belongs to the closure of $\operatorname{Mult}_{1}(A, \|$. \|). On the other hand, by Corollary 1.20 in [11], we know that all continuous multiplicative norms of $A$ lie in the closure of $\operatorname{Mult}_{1}(A, \|$. $\|)$. That makes quite exciting the question whether $\operatorname{Mult}_{1}(A,\|\|$.$) is dense in the whole set \operatorname{Mult}(A, \|$. $\|)$. In order to examine a bit better that question, let us recall that we know a kind of continuous multiplicative semi-norms whose kernel is neither $\{0\}$ nor a maximal ideal: they are due to J. Araujo [1] and are defined in the following way.

Let $r \in] 0,1\left[\right.$ and let $\left(a_{n}\right)_{n \in \mathbb{N}}$ be a sequence in $D$ such that $\lim _{n \rightarrow+\infty}\left|a_{n}\right|=1$. Let $\mathcal{U}$ be an ultrafilter on $\mathbb{N}$ and take $f \in A$. The image of $\mathcal{U}$ by the mapping $h_{f}$ defined on $\mathbb{N}$ as $h_{f}(n)=\|f\|_{\left(d\left(a_{n}, r^{-}\right.\right.}$is included in $[0,\|f\|]$ and therefore that image defines an ultrafilter that converges to a value $\psi(f) \in[0,\|f\|]$. Then, $\psi$ belongs to $\operatorname{Mult}(A, \|$. $\|)$ and $\operatorname{Ker}(\psi)$ is the prime ideal of functions $f \in A$ such that $\lim _{\mathcal{U}}\|f\|_{\left(d\left(a_{n}, r^{-}\right.\right.}=0$.

Definition: We will call Araujo's semi-norms the semi-norms defined in that way.

Theorem 2: $\quad$ Every Araujo's semi-norm lies in the closure of $\operatorname{Mult}_{1}(A, \|$. \|). 
Thus, Theorem 2 strongly suggests that $\operatorname{Mult}_{1}(H(D), \|$. \|) might be dense inside $\operatorname{Mult}(H(D), \|$. $\|)$ : it would just suffice to prove that all continuous multiplicative seminorm of $H(D)$ either are norms, or have a maximal ideal for kernel, or are Araujo's semi-norms, or some semi-norms of the same kind. Unfortunately, we have no mean to prove this.

\section{The Proofs.}

By Theorems 23.5 and 23.6 in [8] we have Lemma 1:

Lemma 1: Let $a \in \mathbb{K}$ and $r>0$ and let $f \in H(d(a, r))$ (resp. $f \in H\left(d\left(a, r^{-}\right)\right.$), resp. $f \in H(C(a, r))))$. If $f$ has no zero in $d(a, r)$, (resp. in $d\left(a, r^{-}\right)$, resp. in $\left.C(a, r)\right)$, $|f(x)|$ is constant. The set of zeros of $f$ in $d(a, r)$ (resp. in $\left(d\left(a, r^{-}\right)\right.$, resp. in $\left.C(a, r)\right)$ is finite.

By Theorem 13.3 and Corollary 13.4 in [8], we can derive Lemma 2:

Lemma 2 : An element of $A$ is quasi-invertible if and only if it is of the form $\mathrm{Pg}$ with $P \in \mathbb{K}[x], P \neq 0$, having all its zeros in $D$ and $g$ an invertible element of $A$.

Lemma 3 is Theorem 20.2 in [8]:

Lemma 3: Let $a \in \mathbb{K}$ and $r>0$ and $b \in d(a, r)$. Then $\varphi_{a, r}=\varphi_{b, r}$.

Proposition 4 due to M. Lazard comes from [16]:

Proposition 4: Suppose $\mathbb{K}$ is spherically complete. Let $a \in \mathbb{K}, R>0$, let $\left(a_{n}\right)_{n \in \mathbb{N}}$ be a sequence of $d\left(a, R^{-}\right)$such that $\lim _{n \rightarrow+\infty}\left|a_{n}-a\right|=R$ and let $\left(q_{n}\right)_{n \in \mathbb{N}}$ be a sequence of integers. There exists $f \in \mathcal{A}\left(d\left(a, R^{-}\right)\right)$admitting each $a_{n}$ as a zero of order $q_{n}$ and having no other zero.

Notation: Let $\widehat{\mathbb{K}}$ be an extension of $\mathbb{K}$ provided with an ultrametric absolute value extending that of $\mathbb{K}$, let $a \in \widehat{\mathbb{K}}$ and let $r>0$. We put $\widehat{d}(a, r)=\{x \in \widehat{\mathbb{K}}|| x-a \mid \leq$ $r\}, \widehat{d}\left(a, r^{-}\right)=\{x \in \widehat{\mathbb{K}}|| x-a \mid<r\}, \widehat{C}(a, r)=\{x \in \widehat{\mathbb{K}}|| x-a \mid=r\}$.

By Theorem 23.1 in [8] we have the following:

Proposition 5: Let $\widehat{\mathbb{K}}$ be an algebraically closed complete extension of $\mathbb{K}, a \in \mathbb{K}$ and $r>0$ and let $f \in H(d(a, r))$ (resp. $f \in H\left(d\left(a, r^{-}\right)\right.$), resp. $f \in H(C(a, r))$ ). The zeros of $f$ in $d(a, r)$ (resp. in $d\left(a, r^{-}\right)$, resp. in $C(a, r)$ ) are the same as in $\widehat{d}(a, r)$ (resp. in $\widehat{d}\left(a, r^{-}\right)$, resp in $\left.\widehat{C}(a, r)\right)$.

From the classical Krasner Mittag-Leffler Theorem ([15] and Theorem 15.1 in [8]), here we can state Proposition 6. 
Proposition 6: Let $E$ be a set of the form $d(0, R) \backslash \bigcup_{i \in J} d\left(a_{i}, r_{i}^{-}\right)$(where $J$ is a set of indices). Then any element $h \in H(E)$ has a unique Mittag-Leffler decomposition of the form $\sum_{n=0}^{\infty} h_{n}$ whereas $h_{0} \in H(d(0, R))$ and for each $n \geq 1, h_{n} \in H\left(K \backslash d\left(a_{i_{n}}, r_{i_{n}}^{-}\right)\right)$and $\lim _{|x| \rightarrow+\infty} h_{n}(x)=0$. Then $\|h\|_{E}=\max \left(\left\|h_{0}\right\|_{d(0, R)}, \sup _{n \geq 1}\left(\left\|h_{n}\right\|_{K \backslash\left(d\left(a_{i_{n}}, r_{i_{n}}^{-}\right)\right.}\right)\right)$. Further, $h_{0}$ is of the form $\sum_{j=0}^{\infty} a_{0, j} x^{j}$ with $\left\|h_{0}\right\|_{d(0, R)}=\sup _{j \geq 0}\left|a_{0, j}\right| R^{j}$ and for $n \geq 1, h_{n}$ is of the form $\sum_{j=1}^{\infty} a_{n, j}\left(x-a_{i_{n}}\right)^{-j}$ with $\left\|h_{n}\right\|_{\mathbb{K} \backslash\left(d\left(a_{i_{n}}, r_{i_{n}}^{-}\right)\right)}=\sup _{j \geq 1}\left|a_{n, j}\right|\left(r_{i_{n}}\right)^{-j}$.

Notation: Let $B$ be a unital commutative $\mathbb{I K}$-algebra. Given $f \in B$, we denote by $s p(f)$ the set of $\lambda \in \mathbb{K}$ such that $f-\lambda$ is not invertible.

By using properties of $T$-filters and particularly idempotent $T$-sequences [8], Lemma 35.1 and Proposition 37.1 (see also [5], Proposition 1.6 and [17]), we have the following proposition:

Proposition 7 : Let $\left(r_{n}\right)_{n \in \mathbb{N}}$ be a sequence in $|\mathbb{K}|$ such that $0<r_{n}<r_{n+1}, \lim _{n \rightarrow+\infty} r_{n}=R$, let $\left(q_{n}\right)_{n \in \mathbb{N}}$ be a sequence of $\mathbb{N}$ such that $q_{n} \leq q_{n+1}$ and $\lim _{n \rightarrow+\infty}\left(\frac{r_{n}}{r_{n+1}}\right)^{q_{n}}=0$. Let $\left.l \in\right] 0, R[$ and for each $n \in \mathbb{N}$, let $b_{n} \in C\left(0,\left(r_{n}\right)^{q_{n}}\right)$, let $a_{n, 1}, \ldots, a_{n, q_{n}}$ be the $q_{n}$-th roots of $b_{n}$ and let $E=d\left(0, R^{-}\right) \backslash\left(\bigcup_{n \in \mathbb{N}}\left(\bigcup_{j=1}^{q_{n}} d\left(a_{n, j}, l^{-}\right)\right)\right.$. Set $f_{n}(x)=\prod_{k=1}^{n} \prod_{j=1}^{q_{k}}\left(\frac{1}{1-\frac{x}{a_{k, j}}}\right)$. Then each $f_{n}$ belongs to $R(E)$ and the sequence $\left(f_{n}\right)_{n \in \mathbb{N}}$ converges in $H(E)$ to an element $f$ strictly vanishing along the pierced increasing filter of center 0 and diameter $R$.

Proposition 8: Let $(B, \|$. $\|)$ be a commutative unital ultrametric $\mathbb{K}$-Banach algebra. Suppose there exist $\ell \in B, \phi, \psi \in \operatorname{Mult}(B, \|$. $\|)$ such that $\psi(\ell)<\phi(\ell), s p(\ell) \cap$ $\Gamma(0, \psi(\ell), \phi(\ell))=\emptyset$ and there exists $\epsilon \in] 0, \phi(\ell)-\psi(\ell)\left[\right.$ satisfying further $\left\|(\ell-a)^{-1}\right\| \leq$ $M, \forall a \in \Gamma(0, \psi(\ell), \phi(\ell)-\epsilon)$. Then there exists $f \in B$ such that $\psi(f)=1, \phi(f)=0$.

Proof: Let $s=\psi(\ell), t=\phi(\ell), Q=\|\ell\|, R=t-\epsilon$ and $l=\frac{1}{M}$. Let $\left.r_{0} \in\right] s, t-\epsilon[$.

Consider the sequence $\left(a_{n, j}\right)_{n \in \mathbb{N}, 1 \leq j \leq q_{n}}$ defined in Proposition 7 and the set

$E=d\left(0, Q^{-}\right) \backslash\left(\bigcup_{n \in \mathbb{N}}\left(\bigcup_{j=1}^{q_{n}} d\left(a_{n, j}, l^{-}\right)\right)\right)$. Then in $H(E)$ we have

$$
\left\|\frac{1}{x-b}\right\|_{E} \leq l \forall b \in \bigcup_{n \in \mathbb{N}}\left(\bigcup_{j=1}^{q_{n}} d\left(a_{n, j}, l^{-}\right)\right) .
$$


There exists a natural homomorphism $\sigma$ from $R(E)$ into $B$ such that $\sigma(x)=\ell$. Since $Q=\|\ell\|$ and $\left\|(\ell-b)^{-1}\right\| \leq M \forall b \in \Gamma(0, s, t)$, by Proposition 15.1 in [8] and by (1) $\sigma$ is clearly continuous with respect to the norms $\|\cdot\|_{E}$ of $R(E)$ and $\|$. $\|$ of $B$. Consequently, $\sigma$ has continuation to a continuous homomorphism from $H(E)$ to $B$.

Now, let $\psi^{\prime}=\psi \circ \sigma, \phi^{\prime}=\phi \circ \sigma$. Then both $\phi^{\prime}, \psi^{\prime}$ belong to $\operatorname{Mult}(H(E), \|$. $\|)$ and satisfy $\psi^{\prime}(x)=s, \phi^{\prime}(x)=t-\epsilon$. So, $\psi^{\prime}$ is of the form $\varphi_{\mathcal{F}}$ with $\mathcal{F}$ a circular filter on $E$ secant with $C(0, s)$ and $\phi^{\prime}$ is of the form $\varphi_{\mathcal{G}}$ with $\mathcal{G}$ a circular filter on $E$ secant with $C(0, t)$.

Consider now the function $f$ constructed in Proposition 7 which, by construction, belongs to $H(E)$ and has no zero and no pole in $d\left(0, s^{-}\right)$. Consequently, $|f(x)|=|f(0)|=$ $1 \forall x \in d\left(0, s^{-}\right)$. Moreover, we have $\lim _{\mathcal{G}} f(x)=0$, hence $\phi^{\prime}(f)=0$. Let $g=\sigma(f)$. Then $\psi(g)=\psi^{\prime}(f)=1$ and $\phi(g)=\phi^{\prime}(f)=0$, which ends the proof.

Proposition 9: Let $\mathcal{U}$ be a coroner ultrafilter on $D$, let $f \in A \backslash \mathcal{J}(\mathcal{U})$ be non-invertible in $A$, such that $\|f\| \leq 1$ and let $g \in A, h \in \mathcal{J}(\mathcal{U})$ such that $f g=1+h$. Let $\tau=\varphi_{\mathcal{U}}(f)$, let $\epsilon \in] 0, \tau\left[\right.$ and let $\Lambda=\left\{x \in D|| f(x) g(x)|-1|_{\infty}<\epsilon,|| f(x)|-\tau|_{\infty}<\epsilon\right\}$.

Suppose that there exist a function $\widetilde{h} \in A$ admitting for zeros in $D$ the zeros of $h$ in $D \backslash \Lambda$ and a function $\bar{h} \in A$ admitting for zeros the zeros of $h$ in $\Lambda$, each counting multiplicities, so that $h=\bar{h} \tilde{h}$. Then $|\widetilde{h}(x)|$ has a strictly positive lower bound in $\Lambda$ and $\bar{h}$ belongs to $\mathcal{J}(\mathcal{U})$.

Moreover, there exists $\omega \in] 0, \tau[$ such that $\omega \leq \inf \{\max (|f(x)|,|\bar{h}(x)|) \mid x \in D\}$. Further, for every $a \in d(0,(\tau-\epsilon))$, we have $\omega \leq \inf \{\max (|f(x)-a|,|\bar{h}(x)|) \mid x \in D\}$.

Proof: Let $u \in \Lambda$ and let $s$ be the distance of $u$ from $\mathbb{K} \backslash \Lambda$. So, the disk $d\left(u, s^{-}\right)$is included in $\Lambda$, hence $f g$ has no zero inside this disk. Consequently, $|f(x) g(x)|$ is a constant $b$ in $d\left(u, s^{-}\right)$. Consider the family $F_{u}$ of radii of circles $C(u, r)$, containing at least one zero of $f g$. By Lemma $1 F_{u}$ has no cluster point different from 1 . Consequently, there exists $\rho \geq s$ such that $f g$ admits at least one zero in $C(u, \rho)$ and admits no zero in $d\left(u, \rho^{-}\right)$. Thus, we know that $|f(x) g(x)|$ is a constant $c$ in $d\left(u, \rho^{-}\right)$. But then, at $u$ we see that $b=c$ and therefore $d\left(u, \rho^{-}\right)$is included in $\Lambda$. Hence $\rho=s$ and therefore $f g$ admits at least one zero $\alpha$ in $C(u, s)$. Thus, at $\alpha$ we have $h(\alpha)=-1$. Therefore, in the disk $d\left(\alpha, s^{-}\right)$we can check that $\varphi_{\alpha, s}(h) \geq 1$. But by Lemma $3 \varphi_{\alpha, s}(h)=\varphi_{u, s}(h)$, hence $\varphi_{u, s}(h) \geq 1$.

Now,

$$
\frac{\|h\|}{\varphi_{u, s}(h)}=\frac{\|\widetilde{h}\|}{\varphi_{u, s}(\widetilde{h})} \frac{\|\bar{h}\|}{\varphi_{u, s}(\bar{h})} \geq \frac{\|\widetilde{h}\|}{\varphi_{u, s}(\widetilde{h})} .
$$

Therefore, since $\varphi_{u, s}(h) \geq 1$, we obtain

$$
\frac{\|\widetilde{h}\|}{\varphi_{u, s}(\widetilde{h})} \leq\|h\|
$$


But since by definition $d\left(u, s^{-}\right)$is included in $\Lambda, \widetilde{h}$ has no zero in this disk, hence $|\widetilde{h}(x)|$ is constant and equal to $\varphi_{u, s}(\widetilde{h})$. Consequently, by (1) we obtain $\frac{\|\widetilde{h}\|}{|\widetilde{h}(u)|} \leq\|h\|$ and therefore we have

$$
|\widetilde{h}(u)| \geq \frac{\|\widetilde{h}\|}{\|h\|} \forall u \in \Lambda \text {. }
$$

This shows that $\widetilde{h}$ does not belong to $\mathcal{J}(\mathcal{U})$, hence, $\varphi_{\mathcal{U}}(\widetilde{h}) \neq 0$. Consequently, $\varphi_{\mathcal{U}}(\bar{h})=0$.

Now, by hypothesis, we have $f g-\bar{h} \widetilde{h}=1$. Since both $g, \widetilde{h}$ belong to $A$ and therefore are bounded in $D$, it is obvious that $\inf \{\max (|f(x)|,|\bar{h}(x)|) \mid x \in D\}>0$. So, we may obviously choose $\omega \in] 0, \tau-\epsilon[$ such that

$$
\omega \leq \inf \{\max (|f(x)|,|\bar{h}(x)|) \mid x \in D\}
$$

Let us now show that for every $a \in d(0,(\tau-\epsilon))$, we have $\omega \leq \inf \{\max (|f(x)-a|,|\bar{h}(x)|) \mid x \in D\}$.

Let $\Lambda^{\prime}=\{x \in D|| f(x) \mid \geq \tau-\epsilon\}$ and let $a \in d\left(0,(\tau-\epsilon)^{-}\right)$. When $\beta$ lies in $\Lambda^{\prime}$, we have $|f(\beta)|>|a|$, hence by $(2), \max (|f(\beta)-a|,|\bar{h}(\beta)|) \geq \omega$ because by(2), either $\omega \leq|\bar{h}(\beta)|$, or $\omega \leq|f(\beta)|=|f(\beta)-a|$.

Now, let $\beta$ lie in $D \backslash \Lambda^{\prime}$ and let $t$ be the distance from $\beta$ to $\Lambda^{\prime}$. Since $D \backslash \Lambda^{\prime}$ is open, $t$ is $>0$. Consider $\varphi_{\beta, t}(f)$. Either there exists $\mu \in \Lambda^{\prime}$ such that $|\beta-\mu|=t$ and then $\varphi_{\beta, t}(f) \geq|f(\mu)| \geq \tau-\epsilon$ or there exists a sequence $\left(x_{n}\right)_{n \in \mathbb{N}} \in \Lambda^{\prime}$ such that $\lim _{n \rightarrow+\infty}\left|\beta-x_{n}\right|=t$ and $\left|x_{n}-\beta\right|>t$. Suppose that we are in the second case: there exists a sequence $\left(x_{n}\right)_{n \in \mathbb{N}} \in \Lambda^{\prime}$ such that $\lim _{n \rightarrow+\infty}\left|\beta-x_{n}\right|=t$ and $\left|x_{n}-\beta\right|>t$. Then the sequence is thinner than the circular filter of center $\beta$ and diameter $t$, hence

$$
\lim _{n \rightarrow+\infty}\left|f\left(x_{n}\right)\right|=\varphi_{\beta, t}(f)
$$

hence $\varphi_{\beta, t}(f) \geq \tau-\epsilon$ again. If $f$ has no zero in $d\left(\beta, t^{-}\right)$, then $|f(x)|$ is a constant in that disk, hence of course $\varphi_{\beta, t}(f)<\tau-\epsilon$. a contradiction. Consequently, $f$ must have a zero $\gamma$ in $d\left(\beta, t^{-}\right)$. Therefore, due to $(2)$, we have $|\bar{h}(\gamma)| \geq \omega$. But since by definition, $\Lambda \subset \Lambda^{\prime}$, the zeros of $\bar{h}$ belong to $\Lambda^{\prime}$. And since $d\left(\beta, t^{-}\right) \cap \Lambda^{\prime}=\emptyset$ actually $\bar{h}$ has no zero in $d\left(\beta, t^{-}\right)$. Consequently $|\bar{h}(x)|$ is constant in $d\left(\beta, t^{-}\right)$and hence $|\bar{h}(\beta)| \geq \omega$, which completes the proof.

The following basic lemma is easily checked and is an application of Proposition 10 in [3]:

Lemma 10: Let $S$ be a set and let $E$ be a subset. Let $\mathcal{F}$ be an ultrafilter on $E$. Then the filter $\widetilde{\mathcal{F}}$ on $S$ with base $\mathcal{F}$ is an ultrafiter inducing on $E$ the ultrafilter $\mathcal{F}$. 
Corollary 10.1: Let $S$ be a set and let $E$ be subset of $S$. Let $\mathcal{F}$ be an ultrafilter on $E$ and let $\widehat{\mathcal{F}}=\mathcal{G}$ be the ultrafilter on $S$ having $\mathcal{F}$ as a base of filter. Let $f$ be a function defined on $S$ with values in a compact topological space $T$. Then $\lim _{\mathcal{G}} f(x)=\lim _{\mathcal{F}} f(x)$.

Proof: Suppose that $f$ admits distinct limits on $\mathcal{F}$ and $\mathcal{G}$. Then $\mathcal{F}$ is a basis of a filter on $S$ that is not secant with $\mathcal{G}$, a contradiction since $\mathcal{F}$ is the ultrafilter induced by $\mathcal{G}$ on E.

Proposition 11: Let $\mathcal{M}$ be a non-principal maximal ideal of $A$ and let $\mathcal{U}$ be an ultrafilter on $D$ such that $\mathcal{M}=\mathcal{J}(\mathcal{U})$. Let $f \in A \backslash \mathcal{M}$ satisfy $\|f\|<1$, let $\tau=\varphi_{\mathcal{U}}(f)$ and let $\left.\epsilon \in\right] 0, \tau[$. There exists $c>0$ such that, for every $a \in d(0, \tau-\epsilon)$, there exists $g_{a} \in A$ satisfying $(f-a) g_{a}-1 \in \mathcal{M}$ and $\left\|g_{a}\right\| \leq c$.

Proof: Suppose first that $f$ is invertible in $A$. By Lemma $1|f(x)|$ is a constant and hence is equal to $\tau$. Therefore, $|f(x)-a|=\tau \forall a \in d(0, \tau-\epsilon)$. Consequently, $f-a$ is invertible and its inverse $g_{a}$ satisfies $\left\|g_{a}\right\|=\tau^{-1}$. Thus, we only have to show the claim when $f$ is not invertible.

Since $f$ does not belong to $\mathcal{M}$, we can find $g \in A$ and $h \in \mathcal{M}$ such that $f g=1+h$ with $h \in \mathcal{M}$.

Let $\widehat{\mathbb{K}}$ be an algebraically closed spherically complete extension of $\mathbb{K}$, let $\widehat{D}$ be the disk $\{x \in \widehat{\mathbb{K}}|| x \mid<1\}$. Let $\widehat{A}$ be the algebra of bounded power series converging in $\widehat{D}$ with coefficients in $\widehat{\mathbb{K}}$.

$\mathcal{U}$ makes a basis of a filter $\widehat{\mathcal{U}}$ on $\widehat{D}$ and by definition, $\mathcal{U}$ is the the filter induced by $\widehat{\mathcal{U}}$ on $D$. By Lemma $10, \widehat{\mathcal{U}}$ is an ultrafilter on $\widehat{D}$.

Consider now $f$ as an element of $\widehat{A}$. Then $\widehat{\mathcal{U}}$ defines an element $\psi$ of $\operatorname{Mult}(\widehat{A}, \|$. $\|)$ as $\psi(\ell)=\lim _{\widehat{\mathcal{U}}}|\ell(x)|, \forall \ell \in \widehat{A}$. Consequently, by Corollary $10.1 \tau$ is equal to $\lim _{\mathcal{U}}|f(x)|$. Let

$$
\Lambda=\left\{x \in \widehat{D}|||f(x) g(x)|-\left.1\right|_{\infty}<\epsilon,|| f(x)|-\tau|_{\infty}<\epsilon\right\}
$$

By Proposition 4 we can factorize $h$ in the form $\widetilde{h} \bar{h}$ where $\widetilde{h} \in \widehat{A}$ is a function admitting for zeros in $\widehat{D}$ the zeros of $h$ in $\widehat{D} \backslash \Lambda$ and $\bar{h} \in \widehat{A}$ is a function admitting for zeros the zeros of $h$ in $\Lambda$, each counting multiplicities. Moreover, we can choose $\bar{h}$ so that $\|\bar{h}\|<1$.

Now, in the field $\widehat{\mathbb{K}}$, by Proposition 9, there exists $\omega>0$ such that for every $a \in \widehat{d}(0,(\tau-\epsilon))$, we have $\omega \leq \inf \{\max (|f(x)-a|,|\bar{h}(x)|) \mid x \in \widehat{D}\}$. This implies that $\inf \{\max (|f(x)-a|,|\bar{h}(x)|) \mid x \in D\} \geq \omega \forall a \in \widehat{d}(0, \tau-\epsilon)$. We notice that $\|f-a\|<1$ for every $a \in \widehat{d}(0, \tau-\epsilon)$, so we may apply Theorem B and obtain a bound $b$ only depending on $f$ and $h$ and functions $\ell_{a}, h_{a} \in \widehat{A}$ such that $(f-a) \ell_{a}+\bar{h} h_{a}=1$, with

$$
\left\|\ell_{a}\right\|<b,\left\|h_{a}\right\|<b \forall a \in \widehat{d}(0, \tau-\epsilon)
$$


By hypothesis we have $\lim _{\mathcal{U}} h(x)=0$. Hence by Corollary 10.1, on $\widehat{D}$ we have $\lim _{\widehat{\mathcal{U}}} h(x)=0$. Then, by Proposition 9 we have $\lim _{\widehat{\mathcal{U}}} \bar{h}(x)=0$ hence, on $D$,

$$
\lim _{\mathcal{U}} \bar{h} h_{a}(x)=0 \forall a \in d(0, \tau-\epsilon)
$$

Now, let us fix $a \in d(0, \tau-\epsilon)$. Let $G$ be the closed $\mathbb{K}$-vector subspace of $\widehat{\mathbb{K}}$ (considered as a $\mathbb{K}$-Banach space), linearly generated over $\mathbb{K}$ by 1 and all coefficients of $\ell_{a}$. Take $\eta>0$ such that $(1+\eta) \max \left(\left\|\ell_{a}\right\|,\left\|h_{a}\right\|\right) \leq b$. We notice that $G$ is a $\mathbb{K}$-Banach space of countable type, hence there exists a $\mathbb{K}$-linear mapping $\Xi$ from $G$ to $\mathbb{K K}$ of norm $\leq 1+\eta$, such that $\Xi(1)=1$ [17]. Let $F$ be the closed $\mathbb{K}$-vector subspace of $\widehat{A}$ consisting of all power series with coefficients in $E$. Then $F$ is a $A$-module and $\Xi$ has continuation to a $A$-linear mapping $\widehat{\Xi}$ from $F$ to $A$ defined as $\widehat{\Xi}\left(\sum_{n=0}^{\infty} b_{n} x^{n}\right)=\sum_{n=0}^{\infty} \Xi\left(b_{n}\right) x^{n}$. This mapping $\widehat{\Xi}$ has a norm bounded by $1+\eta$. Set $g_{a}=\widehat{\Xi}\left(\ell_{a}\right)$. Then by $(1)$ we have

$$
\left\|g_{a}\right\| \leq b(1+\eta) \forall a \in d(0, \tau-\epsilon)
$$

On the other hand, by construction, for every $z \in G$, we have $|\widehat{\Xi}(z)| \leq|z|(1+\eta)$ : that holds particularly for elements of $G \cap D$. Now, since $(f-a)\left(l_{a}\right)-\bar{h} h_{a}=1$, for all $x \in D$, we have $l_{a}(x) \in G, f(x)-a \in K$ and hence $\bar{h} h_{a}(x)$ belongs to $G$. Therefore the inequality applies and shows that $\left|\widehat{\Xi}\left(\bar{h} h_{a}\right)(x)\right| \leq\left|\left(\bar{h} h_{a}\right)(x)\right|(1+\eta)$, hence by $(2)$ we can derive $\lim _{\mathcal{U}} \widehat{\Xi}\left(\bar{h} h_{a}\right)(x)=0 \forall a \in d(0, \tau-\epsilon)$. But since $\widehat{\Xi}$ is a $A$-module linear mapping, we have $\widehat{\Xi}\left((f-a) h_{a}-1\right)=(f-a) g_{a}-1$. Consequently, $\lim _{\mathcal{U}}\left|(f(x)-a) g_{a}(x)-1\right|=0 \forall a \in d(0, \tau-\epsilon)$ and hence $(f-a) g_{a}-1$ belongs to $\mathcal{J}(\mathcal{U})$. Putting $c=b(1+\eta)$, by $(3)$ we are done.

Proof of Theorem 1: Suppose that $A$ is not multbijective and let $\mathcal{M}$ be a maximal ideal which is not univalent. Let $\mathbb{F}$ be the quotient field $\frac{A}{\mathcal{M}}$, let $\theta$ be the canonical surjection from $A$ onto $\mathbb{F}$ and let \|\|$_{q}$ be the $\mathbb{K}$-Banach algebra quotient norm of $F$. By Theorem $\mathrm{C}$ there exists an ultrafilter $\mathcal{U}$ on $D$ such that $\mathcal{M}=\mathcal{J}(\mathcal{U})$. Thus, there exists $\psi \in \operatorname{Mult}(A, \|$. $\|)$ such that $\operatorname{Ker}(\psi)=\mathcal{M}$ and $\psi \neq \varphi_{\mathcal{U}}$. Consequently, there exists $f \in A$ such that $\psi(f) \neq \varphi_{\mathcal{U}}(f)$, with $\psi(f) \neq 0, \varphi_{\mathcal{U}}(f) \neq 0$. We shall check that we may also assume $\psi(f)<\varphi_{\mathcal{U}}(f)$. Indeed, suppose $\psi(f)>\varphi_{\mathcal{U}}(f)$. Let $g \in A$ be such that $\theta(g)=\theta(f)^{-1}$. Then we can see that $\psi(g)=\psi(f)^{-1}, \varphi_{\mathcal{U}}(g)=\left(\varphi_{\mathcal{U}}(f)\right)^{-1}$, therefore $\psi(g)<\varphi_{\mathcal{U}}(g)$. Thus, we may assume $\psi(f)<\varphi_{\mathcal{U}}(f)$ without loss of generality. Similarly, we may obviously assume that $\|f\|<1$.

By construction, $\varphi_{\mathcal{U}}$ factorizes in the form $\phi_{1} \circ \theta$ and similarly, $\psi$ factorizes as $\phi_{2} \circ \theta$ with $\phi_{1}, \phi_{2} \in \operatorname{Mult}\left(\mathbb{F}, \|\right.$. $\left.\|_{q}\right)$. So, on $F$ we have $\phi_{1}(\theta(f))>\phi_{2}(\theta(f))$. 
Let $\tau=\varphi_{\mathcal{U}}(f)$ and let $\left.\epsilon \in\right] 0, \tau[$. By Proposition 11, there exists $c>0$ such that, for every $a \in d(0, \tau-\epsilon)$, there exists $g_{a} \in A$ satisfying $(f-a) g_{a}-1 \in \mathcal{M}$ and $\left\|g_{a}\right\| \leq c$. Now, $\theta\left(g_{a}\right)=(\theta(f-a))^{-1}$. Thus, $\left\|(\theta(f-a))^{-1}\right\|_{q} \leq c$ for all $a \in d(0, \tau-\epsilon)$. Therefore, by applying Proposition 8 to the $\mathbb{K}$-Banach algebra $\mathbb{F}$, we can see that there exists $y \in \mathbb{F}$ such that $\phi_{1}(y)=1, \phi_{2}(y)=0$. Therefore, taking $g \in A$ such that $\theta(g)=y$, we get $\varphi_{\mathcal{U}}(g)=0, \psi(g)=1$, a contradiction to the hypothesis $\operatorname{Ker}\left(\varphi_{\mathcal{U}}\right)=\operatorname{Ker}(\psi)$. This finishes the proof that $A$ is multbijective.

Proof of Theorem 2: Given a $\phi \in \operatorname{Mult}(A, \|$. $\|), \epsilon>0$ and $f_{1}, \ldots, f_{q} \in A$, we set $\mathcal{W}\left(\psi, f_{1}, \ldots, f_{q}, \epsilon\right)=\left\{\theta \in \operatorname{Mult}(A,\|\|)||. \phi\left(f_{j}\right)-\left.\theta\left(f_{j}\right)\right|_{\infty} \leq \epsilon \forall j=1, \ldots, q\right.$. We know that such sets make a basis of neighborhoods of $\phi$ with respect to the topology of $\operatorname{Mult}(A,\|\|$.$) .$

Now, let $\psi$ be an Araujo semi-norm defined by a sequence of disks $d\left(a_{n}, r\right)$, with $\lim _{n \rightarrow+\infty}\left|a_{n}\right|=1$ and an ultrafilter $\mathcal{T}$ on $\mathbb{N}$ so that $\psi(f)=\lim _{\mathcal{T}}\left|f\left(a_{n}\right)\right| \forall f \in A$.

Consider a neighborhood $\mathcal{W}\left(\psi, f_{1}, \ldots, f_{q}, \epsilon\right)$ of $\psi$, with $f_{j} \in A$ and $\epsilon>0$. Set $s_{j}=$ $\psi\left(f_{j}\right), j=1, \ldots, q$. By hypothesis, there exists an infinite subset $S \in \mathcal{T}$ such that $\mid \varphi_{a_{n}, r}-$ $\left.s_{j}\right|_{\infty} \leq \epsilon \forall n \in T, \forall j=1, \ldots q$.

Let us fix $m \in S$. For each $j=1, \ldots q$, we then have

$$
\lim _{\left|x-a_{m}\right| \rightarrow r}\left|f_{j}(x)\right|=\left\|f_{j}\right\|_{d\left(a_{m}, r\right)}=\varphi_{a_{m}, r}\left(f_{j}\right)
$$

therefore there exists $b_{m} \in d\left(a_{m}, r\right)$ such that ||$f_{j}\left(b_{m}\right)\left|-\varphi_{a_{m}, r}\left(f_{j}\right)\right|_{\infty} \leq \epsilon \forall j=1, \ldots, q$ and therefore we derive $\left|\varphi_{b_{m}}\left(f_{j}\right)-\psi\left(f_{j}\right)\right|_{\infty} \leq 2 \epsilon \forall j=1, \ldots, q$. Consequently, $\varphi_{b_{m}}$ belongs to $\mathcal{W}\left(\psi, f_{1}, \ldots, f_{q}, 2 \epsilon\right)$, which proves that $\psi$ belongs to the closure of $\operatorname{Mult}_{1}(A, \|$. $\|)$.

Acknowledgements: I am very grateful to Jean-Paul Bézivin and to the anonymous referee for carefully reading this paper and noticing many misprints and abstraction errors. I am particularly grateful to the referee who pointed out to me improvements and corrections in the proof of Proposition 11.

\section{References}

[1] Araujo J. Prime and maximals ideals in the spectrum of the ultrametric algebra $H^{\infty}(D)$, preprint.

[2] Berkovich, V. Spectral Theory and Analytic Geometry over Non-archimedean Fields. AMS Surveys and Monographs 33, (1990).

[3] Bourbaki, N. Topologie générale, Ch. I, Actualités scientifiques et industrielles, Hermann, Paris (1981).

[4] Carleson, L. Interpolation by bounded analytic functions and the corona problem. Annals of Math. 76, p. 547-559 (1962).

[5] Escassut, A. T-filtres, ensembles analytiques et transformations de Fourier padique. Annales de l'Institut Fourier, tome 25, fasc 2, pp 35-80 (1975). 
[6] Escassut, A. Spectre maximal d'une algèbre de Krasner, Colloquium Mathematicum, XXXVIII, fasc. 2, p. 339-357, (1978).

[7] Escassut, A. The ultrametric spectral theory, Periodica Mathematica Hungarica, Vol.11, (1), p7-60, (1980).

[8] Escassut, A. Analytic Elements in p-adic Analysis, World Scientific Publishing Inc., Singapore (1995).

[9] Escassut, A. Ultrametric Banach Algebras, World Scientific Publishing Inc., Singapore (2003).

[10] Escassut, A. and Mainetti, N. About the ultrametric Corona problem Bulletin des Sciences Mathématiques 132, p. 382-394 (2008)

[11] Escassut, A. Ultrametric Corona problem and spherically complete fields, Proceedings of the Edingburgh Mathematical Society, (Series 2), Volume 53, Issue 02, pp 353-371 (2010).

[12] Garandel, G. Les semi-normes multiplicatives sur les algèbres d'éléments analytiques au sens de Krasner, Indag. Math., 37, n4, p.327-341, (1975).

[13] Guennebaud, B. Algèbres localement convexes sur les corps valués, Bull. Sci. Math. 91, p. 75-96, (1967).

[14] Guennebaud, B. Sur une notion de spectre pour les algèbres normées ultramétriques, thèse Université de Poitiers, (1973).

[15] Krasner, M. Prolongement analytique uniforme et multiforme dans les corps valués complets. Les tendances géométriques en algèbre et théorie des nombres, ClermontFerrand, p.94-141 (1964). Centre National de la Recherche Scientifique (1966), (Colloques internationaux du C.N.R.S. Paris, 143).

[16] Lazard, M. Les zéros des fonctions analytiques sur un corps valué complet, IHES, Publications Mathématiques n14, p.47-75 ( 1962).

[17] Sarmant, M.-C. and Escassut, A. T-suites idempotentes, Bull. Sci. Math. 106, p.289-303, (1982).

[18] K. Shamseddine and M. Berz. Analysis on the Levi-Civita field. A brief overview. Advances in $p$-adic and Non-Archimedean Analysis, Contemporary Mathematics 508, (2010).

[19] Van Der Put, M. The Non-Archimedean Corona Problem Table Ronde Anal. non Archimedienne, Bull. Soc. Math. Mémoire 39-40, p. 287-317 (1974).

Alain Escassut

Laboratoire de Mathématiques UMR 6620

Université Blaise Pascal

(Clermont-Ferrand)

Les Cézeaux

63178 AUBIERE CEDEX

FRANCE

Alain.Escassut@math.univ-bpclermont.fr 